doi:10.13108/2015-7-4-58

UDC 517.518.6

\title{
VARIOUS DEFINITIONS OF SPECTRUM OF ALMOST PERIODIC FUNCTIONS
}

\author{
N.P. GIRYA, S.YU. FAVOROV
}

\begin{abstract}
We consider various definitions of the spectrum for almost periodic functions in a finite dimensional space for uniform, Stepanov's, Weil's, Besicovitch's metrics. We prove that in these cases the classical definition of spectrum is equivalent to an analogue of definition of Beurling's spectrum.
\end{abstract}

Keywords: almost periodic function, spectrum, Stepanov's metric, Weil's metric, Besicovitch's metric, Beurling's spectrum.

Mathematics Subject Classification: 42A75, 30B50

\section{INTRODUCTION}

Let $f$ be a bounded measurable function on the real axis $\mathbb{R}$. Its Beurling's spectrum is the set of $\lambda \in \mathbb{R}$ such that $e^{i \lambda t}$ lies in the closure of finite sums $\sum_{j} c_{j} f\left(t+x_{j}\right)$ and the closure is understood in the sense of the weak topology in space $L^{\infty}(\mathbb{R})$ as dual for $L^{1}(\mathbb{R})([2])$. It can be shown that Beurling's spectrum introduced in such way is a closed set and if $f \in L^{\infty}(\mathbb{R}) \cap L^{1}(\mathbb{R})$, this set coincides with the support of the Fourier transform $\widehat{f}(\lambda)$ of function $f(t)$.

If $f(t)$ is an almost periodic (a.p.) function on the real axis, its spectrum is usually defined by the identity

$$
\operatorname{sp} f=\left\{\lambda \in \mathbb{R}: a(\lambda, f)=\lim _{T \rightarrow \infty} \frac{1}{2 T} \int_{-T}^{T} f(t) e^{-i \lambda t} \neq 0\right\} .
$$

This spectrum can be any countable set and this is why it does not necessarily coincides with Beurling's spectrum of function $f$. However, as it was shown in [4], if Beurling's spectrum of function $f$ is bounded and countable or only countable (if $f$ is uniformly continuous), then $f$ is an a.p. function.

In the present work we consider an analogue of Beurling's spectrum employing stronger topologies. We show that such Beurling's spectrum coincides with the classical definition of the spectrum for an almost periodic function $f$. We consider functions in space $\mathbb{R}^{n}, n \geqslant 1$, being almost periodic both in the sense of Bohr, Stepanov, Weil, Besicovitch. We notice that in [3], [7], 8] we studied the properties of spectrum of similar functions and its relations with analytic continuation of function on space $\mathbb{C}^{n}$.

Let us formulate the definitions and theorems in the theory of a.p. functions, which we shall employ in what follows.

By $\Omega(x, a)$ we denote a squared beam, i.e., the Cartesian product of segments

$$
\Omega(x, a):=\left[x_{1}, x_{1}+a_{1}\right] \times \ldots \times\left[x_{n}, x_{n}+a_{n}\right],
$$

where $x, a \in \mathbb{R}^{n}, x=\left(x_{1}, \ldots, x_{n}\right), a=\left(a_{1}, \ldots, a_{n}\right)$.

N.P. Girya, S.Yu. Favorov, Various definitions of SPECtrum of almost Periodic functions.

(C) Girya N.P., FAVOROV S.Yu. 2015.

Submitted August 25, 2015. 
We introduce the following definitions of the distance between two functions $f(x)$ and $g(x)$ such that $f: \mathbb{R}^{n} \rightarrow \mathbb{C}, g: \mathbb{R}^{n} \rightarrow \mathbb{C}$.

Definition 1. The quantity

$$
D_{U}[f(x), g(x)]=\sup _{x \in \mathbb{R}^{n}}|f(x)-g(x)|
$$

is called the distance in the uniform metric.

Definition 2. (see [9]) The quantity

$$
D_{S_{l}^{p}}[f(x), g(x)]=\sup _{x \in \mathbb{R}^{n}}\left[\frac{1}{l^{n}} \int_{\Omega(x, l I)}|f(y)-g(y)|^{p} d y\right]^{\frac{1}{p}},
$$

where $I=(1, \ldots, 1)$ is called $S$-distance of order $p(p \geqslant 1)$ associated with length $l(l>0)$. This metric is called Stepanov's metric.

In the case $l=1$, instead of $D_{S_{1}^{p}}$ we shall write $D_{S^{p}}$. We note that $S$-distances are equivalent for various $l$ (for the case of one variable see [9]; in the multi-dimensional case the proof is similar).

Definition 3. (see [9]) The quantity

$$
D_{W^{p}}[f(x), g(x)]=\lim _{l \rightarrow \infty} D_{S_{l}^{p}}[f(x), g(x)]=\lim _{l \rightarrow \infty} \sup _{x \in \mathbb{R}^{n}}\left[\frac{1}{l^{n}} \int_{\Omega(x, l I)}|f(y)-g(y)|^{p} d y\right]^{\frac{1}{p}}
$$

is called $W$-distance of order $p,(p \geqslant 1)$. Such metric is called Weil's metric.

Definition 4. (see [1]) The quantity

$$
D_{B^{p}}[f(x), g(x)]=\left\{\lim _{T \rightarrow \infty} \frac{1}{(2 T)^{n}} \int_{\Omega(-T I, 2 T I)}|f(y)-g(y)|^{p} d y\right\}^{\frac{1}{p}}=\left\{\bar{M}\left\{|f-g|^{p}\right\}\right\}^{\frac{1}{p}}
$$

$(p \geqslant 1)$ is called Besicovitch's distance of order $p$. Such metric is called Besicovitch's metric.

In Definition 1 we always assume that functions $f(x)$ and $g(x)$ are continuous and bounded. In Definitions 2-4 functions $f(x)$ and $g(x)$ are measurable and $p$-th power integrable in each compact set.

Let $D[f(x), g(x)]$ be one of the above mentioned metrics $D_{U}, D_{S_{l}^{p}}, D_{W^{p}}, D_{B^{p}}$.

Definition 5. (see [1]) Function $f(x): \mathbb{R}^{n} \rightarrow \mathbb{C}$ is called D-almost periodic function if there exists a sequence of finite exponential sums $P_{n}(x)=\sum_{j} c_{j} e^{i\left\langle\lambda_{j}, x\right\rangle}, c_{j} \in \mathbb{C}, \lambda_{j} \in \mathbb{R}^{n}$, such that

$$
\lim _{n \rightarrow \infty} D\left[f(x), P_{n}(x)\right]=0 .
$$

We note that one often uses equivalent definitions of metrics $D_{U}, D_{S_{l}^{p}}, D_{W^{p}}$ in terms of almost periods.

Definition 6. A vector $\tau \in \mathbb{R}^{n}$ is called $(D, \varepsilon)$-almost period of a p-th power integrable (in each compact set) function $f(x): \mathbb{R}^{n} \rightarrow \mathbb{C}$ if the inequality

$$
D[f(x+\tau), f(x)]<\varepsilon
$$

holds true.

Definition 7. A measurable and $p$-th power integrable (in each compact set) function $f(x)$ : $\mathbb{R}^{n} \rightarrow \mathbb{C}$ is called $D$-almost periodic $\left(D=D_{S_{p}^{l}}\right)$ if for each $\varepsilon>0$ there exists a relatively dense set of $E(D, \varepsilon)$-almost periods of $f(x)$. At that, a set $E \subset \mathbb{R}^{n}$ is called relatively dense if there exists $L<\infty$ such that $E \cap \Omega[a, L I] \neq \emptyset$ for each $a \in \mathbb{R}^{n}$. 
For $W^{p}$-a.p. functions this definition should be slightly changed. Under the above conditions, $f$ is $W^{p}$-a.p. function if for each $\varepsilon>0$ there exist $l<\infty$ and relatively dense set of $E\left(D_{S_{p}^{l}}, \varepsilon\right)$ almost periods $f(x)$.

For $U$-a.p. function one should replace the property of measurability and integrability by the property of continuity of function $f(x)$.

The definition of a.p. function implies immediately

Theorem 1. D-a.p. function $f(x): \mathbb{R}^{n} \rightarrow \mathbb{C}$ is D-bounded and D-uniformly continuous.

Theorem 2. (see [9]) For each $D$-a.p. function $f(x)$ of several variables there exists the mean value

$$
M\{f(x)\}=\lim _{T \rightarrow \infty} \frac{1}{T^{n}} \int_{\Omega(0, T I)} f(x) d x .
$$

The limit $\lim _{T \rightarrow \infty} \frac{1}{T^{n}} \int_{\Omega(a, T I)} f(x) d x=M\{f(x+a)\}$ exists uniformly in $a=\left(a_{1}, \ldots, a_{n}\right) \in \mathbb{R}^{n}$, and the identity

$$
M\{f(x+a)\}=M\{f(x)\}
$$

holds true. The uniformity is absent in the case $D=D_{B^{p}}$, although the identity is still valid.

In particular, $M\{f(x)\}=\lim _{T \rightarrow \infty} \frac{1}{(2 T)^{n}} \int_{\Omega(-T I, 2 T I)} f(x) d x$.

To each $D$-a.p. function $f(x)$ of several variables we associate the Fourier series $f(x) \sim$ $\sum_{\lambda \in \mathbb{R}^{n}} a(\lambda, f) e^{i\langle\lambda, x\rangle}$, where $a(\lambda, f)=M\left\{f(x) e^{-i\langle\lambda, x\rangle}\right\}$.

Definition 8. (see [1] for $n=1$ and [5] for $n>1$ ) The spectrum of function $f(x)$ is the set $\operatorname{sp} f=\left\{\lambda \in \mathbb{R}^{n}: a(\lambda, f) \neq 0\right\}$.

Theorem 3. The spectrum of a D-a.p. function $f(x)$ of several variables is at most countable.

The proofs of Theorems 1-3 for the one-dimensional case can be found in [9], while the case of several variables can be treated in the same way.

Definition 9. Beurling's type spectrum of an a.p. function is

$$
\operatorname{sp}_{B} f=\left\{\lambda \in \mathbb{R}^{n}: e^{i\langle\lambda, t\rangle} \in \overline{\operatorname{Lin}\{f(x+t)\}_{x \in \mathbb{R}^{n}}}\right\} .
$$

We note that the closure is taken in the same metric $D$, in which we define the periodicity.

In the work we prove the following theorem.

Theorem 4. Let $f(x): \mathbb{R}^{n} \rightarrow \mathbb{C}$ be an D-a.p. function integrable in the Riemann sense over each squared beam. Then $\operatorname{sp} f=\operatorname{sp}_{B} f$.

Proof. Let us prove the inclusion $\operatorname{sp} f \subset \mathrm{sp}_{B} f$.

1) Consider the case $D=D_{W^{p}}$.

Let $\lambda \in \operatorname{sp} f$, then $M\left\{f(t) e^{-i\langle\lambda, t\rangle}\right\}=\alpha \neq 0$. We let $f_{1}(t)=\frac{1}{\alpha} f(t) e^{-i\langle\lambda, t\rangle}$. It means that there exists the limit

$$
\lim _{T \rightarrow \infty} \frac{1}{T^{n}} \int_{\Omega(0, T I)} f_{1}(x+t) d x=1
$$

uniformly in parameter $t \in \mathbb{R}^{n}$. Thus, for each $\varepsilon>0$ there exists an independent of $t$ number $T_{0}=T_{0}(\varepsilon)$ such that for each $T>T_{0}$ the inequality

$$
\left|\frac{1}{T^{n}} \int_{\Omega(0, T I)} f_{1}(x+t) d x-1\right|<\varepsilon
$$


holds true. Let us show that for each $\varepsilon>0$ there exists an integral sum associated with the above written integral and approximating it in Weil's metric uniformly in $t \in \mathbb{R}^{n}$. Since $f_{1}(t)$ is $W^{p}$-a.p. function, it is $W^{p}$-uniformly continuous, i.e., for each $\varepsilon>0$ there exist $l_{0}=l_{0}(\varepsilon)$ and $\delta=\delta(\varepsilon)$ such that for $l>l_{0}$ and $|h|<\delta$ we have

$$
D_{S_{l}^{p}}\left[f_{1}(t+h), f_{1}(t)\right]<\varepsilon .
$$

Let $\left\{B_{k}\right\}_{k=1}^{N}$ be an arbitrary partition of squared beam $\Omega(0, T I)$ such that $\operatorname{diam}\left(\mathrm{B}_{\mathrm{k}}\right)<\delta$ $(k=\overline{1, N})$, where $\delta$ is taken from 2 . It is obvious that $\sum_{k=1}^{N} \mu\left(B_{k}\right)=T^{n}$ (where $\mu$ is the Lebesgue measure in $\mathbb{R}^{n}$ ). Let $\left\{h_{k}\right\}_{k=1}^{N}$ be an arbtirary set of points in $\mathbb{R}^{n}$ such that $h_{k} \in B_{k}$, $k=\overline{1, N}$. Then the integral sum corresponding to this partition reads as

$$
\sigma\left(f_{1}\right)=\frac{1}{T^{n}} \sum_{k=1}^{N} f_{1}\left(t+h_{k}\right) \mu\left(B_{k}\right)
$$

Let us show that

$$
D_{W^{p}}\left[\frac{1}{T^{n}} \int_{\Omega(0, T I)} f_{1}(x+t) d x, \sigma\left(f_{1}\right)\right]<\varepsilon
$$

i.e., that

$$
\lim _{l \rightarrow \infty} D_{S_{l}^{p}}\left[\frac{1}{T^{n}} \int_{\Omega(0, T I)} f_{1}(x+t) d x, \sigma\left(f_{1}\right)\right]<\varepsilon .
$$

In other words, there exists $l_{0}>0$ such that for each $l>l_{0}$ and $y \in \mathbb{R}^{n}$ the inequality

$$
\left(\frac{1}{l^{n}} \int_{\Omega(0, l I)}\left|\frac{1}{T^{n}} \int_{\Omega(0, T I)} f_{1}(x+y+t) d x-\frac{1}{T^{n}} \sum_{k=1}^{N} f_{1}\left(t+h_{k}+y\right) \mu\left(B_{k}\right)\right|^{p} d t\right)^{\frac{1}{p}}<\varepsilon
$$

or

$$
\left(\frac{1}{l^{n}} \int_{\Omega(0, l I)}\left|\frac{1}{T^{n}} \sum_{k=1}^{N} \int_{B_{k}}\left(f_{1}(x+t+y)-f_{1}\left(t+h_{k}+y\right)\right) d x\right|^{p} d t\right)^{\frac{1}{p}}<\varepsilon .
$$

We apply Hölder inequality to the expression

$$
\left|\frac{1}{T^{n}} \sum_{k=1}^{N} \int_{B_{k}}\left(f_{1}(x+t+y)-f_{1}\left(t+h_{k}+y\right)\right) d x\right|^{p}
$$

and obtain that it does not exceed

$$
\frac{1}{T^{n}} \sum_{k=1}^{N} \int_{B_{k}}\left|f_{1}(x+t+y)-f_{1}\left(t+h_{k}+y\right)\right|^{p} d x .
$$

To prove (4), it is sufficient to satisfy

$$
\frac{1}{l^{n}} \int_{\Omega(0, l I)} \frac{1}{T^{n}} \sum_{k=1}^{N} \int_{B_{k}}\left|f_{1}(x+t+y)-f_{1}\left(t+h_{k}+y\right)\right|^{p} d x d t<\varepsilon^{p} .
$$

Switching the integration order, we obtain

$$
\frac{1}{T^{n}} \sum_{k=1}^{N} \int_{B_{k}} \frac{1}{l^{n}} \int_{\Omega(0, l I)}\left|f_{1}(x+t+y)-f_{1}\left(t+h_{k}+y\right)\right|^{p} d t d x .
$$


We note that the following inequality

$$
\begin{gathered}
\frac{1}{T^{n}} \sum_{k=1}^{N} \int_{B_{k}} \frac{1}{l^{n}} \int_{\Omega(0, l I)}\left|f_{1}(t+x+y)-f_{1}\left(t+h_{k}+y\right)\right|^{p} d t d x \\
\leqslant \frac{1}{T^{n}} \sum_{k=1}^{N} \int_{B_{k}} D_{S_{l}^{p}}^{p}\left[f_{1}(t+x), f_{1}\left(t+h_{k}\right)\right] d x
\end{gathered}
$$

holds true. We apply inequality (2). Since $\mu\left(B_{k}\right)<\delta, k=\overline{1, N}$, then $\left|(x+t)-\left(t+h_{k}\right)\right|<\delta$ holds true $\forall x \in B_{k}$. Hence, there exists $l_{0}>0$ such that for each $l>l_{0}$, each $k=\overline{1, N}$, each $x \in B_{k}$ we have

$$
D_{S_{l}^{p}}\left[f_{1}(t+x), f_{1}\left(t+h_{k}\right)\right]<\varepsilon .
$$

This is why expression (7) does not exceed

$$
\frac{1}{T^{n}} \sum_{k=1}^{N} \int_{B_{k}} \varepsilon^{p} d x=\varepsilon^{p}
$$

that implies (5).

Thus, for each $\varepsilon>0$ there exists integral sum $\sigma\left(f_{1}\right)$ associated with a partition independent of $t \in \mathbb{R}^{n}$ such that

$$
D_{W^{p}}\left[\frac{1}{T^{n}} \int_{\Omega(0, T I)} f_{1}(x+t) d x, \sigma\left(f_{1}\right)\right]<\varepsilon .
$$

Then

$$
D_{W^{p}}\left[\sigma\left(f_{1}\right), 1\right] \leqslant D_{W^{p}}\left[\frac{1}{T^{n}} \int_{\Omega(0, T I)} f_{1}(x+t) d x, \sigma\left(f_{1}\right)\right]+D_{W^{p}}\left[\frac{1}{T^{n}} \int_{\Omega(0, T I)} f_{1}(x+t) d x, 1\right] .
$$

Let us check that

$$
D_{W^{p}}\left[\frac{1}{T^{n}} \int_{\Omega(0, T I)} f_{1}(x+t) d x, 1\right]<\varepsilon .
$$

It follows from inequality (1) that

$$
\left|\frac{1}{T^{n}} \int_{\Omega(0, T I)} f_{1}(x+t) d x-1\right|^{p}<\varepsilon^{p} .
$$

Employing (8), we write out an obvious chain of relations

$$
\begin{aligned}
D_{W^{p}}\left[\frac{1}{T^{n}} \int_{\Omega(0, T I)} f_{1}(x+t) d x, 1\right] & =\lim _{l \rightarrow \infty} \sup _{y \in \mathbb{R}^{n}}\left(\frac{1}{l^{n}} \int_{\Omega(y, l I)}\left|\frac{1}{T^{n}} \int_{\Omega(0, T I)} f_{1}(t+x) d x-1\right|^{p} d t\right)^{\frac{1}{p}} \\
& <\left(\frac{1}{l^{n}} l^{n} \varepsilon^{p}\right)^{\frac{1}{p}}=\varepsilon .
\end{aligned}
$$

It follows that $D_{W^{p}}\left[\sigma\left(f_{1}\right), 1\right]<2 \varepsilon$.

Returning back to the previous notation, we have

$$
D_{W^{p}}\left[\frac{\alpha}{T^{n}} \sum_{k=1}^{N} f\left(t+h_{k}\right) e^{-i\langle\lambda, t\rangle} e^{-i\left\langle\lambda, h_{k}\right\rangle} \mu\left(B_{k}\right), 1\right]<2 \varepsilon .
$$


We let $A_{k}:=\frac{\alpha \mu\left(B_{k}\right) e^{-i\left\langle\lambda, h_{k}\right\rangle}}{T^{n}}$ and we get

$$
D_{W^{p}}\left[\sum_{k=1}^{N} A_{k} f\left(t+h_{k}\right), e^{i\langle\lambda, t\rangle}\right]<2 \varepsilon .
$$

Since $\varepsilon$ is arbitrary, we obtain that $\lambda \in \operatorname{sp}_{B} f$.

2) In the case $D=D_{S^{p}}$, one just reproduce the above proof almost literally. In the parts of the proof involving a limit as $l \rightarrow \infty$, one should choose $l=1$. Since $S$-distances corresponding to various $l$ are topologically equivalent, the inclusion is also proven for $D=D_{S_{l}^{p}}$.

3) We consider the case $D=D_{B^{p}}$.

Let $\lambda \in \operatorname{sp} f$. We denote $f_{1}(t)=\frac{1}{\alpha} f(t) e^{-i\langle\lambda, t\rangle}$, where $\alpha=M\left\{f(t) e^{-i\langle\lambda, t\rangle}\right\} \neq 0$. It means that there exists the limit

$$
\lim _{T \rightarrow \infty} \frac{1}{(2 T)^{n}} \int_{\Omega(-T I, 2 T I)} f_{1}(x+t) d x=1 .
$$

For each $\varepsilon>0$ there exists a number $T_{0}=T_{0}(\varepsilon)$ (depending probably of $t \in \mathbb{R}^{n}$ ) such that for each $T>T_{0}$ the inequality

$$
\left|\frac{1}{(2 T)^{n}} \int_{\Omega(-T I, 2 T I)} f_{1}(x+t) d x-1\right|<\varepsilon
$$

is satisfied. Let us show that for each $\varepsilon>0$ there exists an integral sum associated with the above integral, which approximates the integral in Besicovitch's metric. Since $f_{1}(t)$ is $B^{p}$-a.p. function, it is $B^{p}$-uniformly continuous, i.e., for each $\varepsilon>0$ there exists $\delta=\delta(\varepsilon)$ such that

$$
D_{B^{p}}\left[f_{1}(t+h), f_{1}(t)\right]<\varepsilon
$$

for $|h|<\delta$.

Let $\left\{B_{k}\right\}_{k=1}^{N}$ be an arbitrary partition of squared beam $\Omega(-T I, 2 T I)$ such that $\operatorname{diam}\left(\mathrm{B}_{\mathrm{k}}\right)<\delta$ $(k=\overline{1, N})$, where $\delta$ is taken from 10 . It is obvious that $\sum_{k=1}^{N} \mu\left(B_{k}\right)=(2 T)^{n}$, where $\mu$ is the Lebesgue measure in $\mathbb{R}^{n}$. Let $\left\{h_{k}\right\}_{k=1}^{N}$ be an arbitrary set of points in $\mathbb{R}^{n}$ such that $h_{k} \in B_{k}$, $k=\overline{1, N}$. Then the integral sum associated with such partition reads as

$$
\sigma\left(f_{1}\right)=\frac{1}{(2 T)^{n}} \sum_{k=1}^{N} f_{1}\left(t+h_{k}\right) \mu\left(B_{k}\right) .
$$

Let us show that

$$
D_{B^{p}}\left[\frac{1}{(2 T)^{n}} \int_{\Omega(-T I, 2 T I)} f_{1}(x+t) d x, \sigma\left(f_{1}\right)\right]<\varepsilon,
$$

i.e., there exists $S_{0}>0$ such that for each $S>S_{0}$ the inequality

$$
\left(\frac{1}{(2 S)^{n}} \int_{\Omega(-S I, 2 S I)}\left|\frac{1}{(2 T)^{n}} \int_{\Omega(-T I, 2 T I)} f_{1}(x+t) d x-\frac{1}{(2 T)^{n}} \sum_{k=1}^{N} f_{1}\left(t+h_{k}\right) \mu\left(B_{k}\right)\right|^{p} d t\right)^{\frac{1}{p}}<\varepsilon
$$

holds true or

$$
\left(\frac{1}{(2 S)^{n}} \int_{\Omega(-S I, 2 S I)}\left|\frac{1}{(2 T)^{n}} \sum_{k=1}^{N} \int_{B_{k}}\left(f_{1}(x+t)-f_{1}\left(t+h_{k}\right)\right) d x\right|^{p} d t\right)^{\frac{1}{p}}<\varepsilon .
$$


We apply Hölder inequality to the expression

$$
\left|\frac{1}{(2 T)^{n}} \sum_{k=1}^{N} \int_{B_{k}}\left(f_{1}(x+t)-f_{1}\left(t+h_{k}\right)\right) d x\right|^{p}
$$

and obtain that it does not exceed

$$
\frac{1}{(2 T)^{n}} \sum_{k=1}^{N} \int_{B_{k}}\left|f_{1}(x+t)-f_{1}\left(t+h_{k}\right)\right|^{p} d x .
$$

To satisfy (12), it is sufficient to prove

$$
\frac{1}{(2 S)^{n}} \int_{\Omega(-S I, 2 S I)} \frac{1}{(2 T)^{n}} \sum_{k=1}^{N} \int_{B_{k}}\left|f_{1}(x+t)-f_{1}\left(t+h_{k}\right)\right|^{p} d x d t<\varepsilon^{p}
$$

or

$$
\frac{1}{(2 T)^{n}} \sum_{k=1}^{N} \int_{B_{k}} \frac{1}{(2 S)^{n}} \int_{\Omega(-S I, 2 S I)}\left|f_{1}(x+t)-f_{1}\left(t+h_{k}\right)\right|^{p} d t d x<\varepsilon^{p} .
$$

Let us estimate each term in the sum. Let $\forall x \in B_{k}, \max \left\{|\mathrm{x}|,\left|\mathrm{h}_{\mathrm{k}}\right|\right\}=\mathrm{b}_{\mathrm{k}}$. Then the following chain of inequalities hold:

$$
\begin{aligned}
& \left(\frac{1}{(2 S)^{n}} \int_{\Omega(-S I, 2 S I)}\left|f_{1}(x+t)-f_{1}\left(t+h_{k}\right)\right|^{p} d t\right)^{\frac{1}{p}} \\
& \leqslant\left(\frac{1}{(2 S)^{n}} \int_{\Omega(-S I, 2 S I)}\left|f_{1}(x+t)\right|^{p} d t\right)^{\frac{1}{p}}+\left(\frac{1}{(2 S)^{n}} \int_{\Omega(-S I, 2 S I)}\left|f_{1}\left(t+h_{k}\right)\right|^{p} d t\right)^{\frac{1}{p}} \\
& \leqslant\left(\frac{2^{n+1}\left(S+b_{k}\right)^{n}}{(2 S)^{n}} \frac{1}{2^{n}\left(S+b_{k}\right)^{n}} \int_{\Omega\left(\left(-S-b_{k}\right) I, 2\left(S+b_{k}\right) I\right)}\left|f_{1}(t)\right|^{p} d t\right)^{\frac{1}{p}} .
\end{aligned}
$$

Since $f_{1}(t)$ is an $B^{p}$-a.p. function, for sufficiently large $S$ there exists $C_{k}>0, k=1, \ldots, N$, such that

$$
\left(\frac{1}{2^{n}\left(S+b_{k}\right)^{n}} \int_{\Omega\left(\left(-S-b_{k}\right) I, 2\left(S+b_{k}\right) I\right)}\left|f_{1}(t)\right|^{p} d t\right)^{\frac{1}{p}}<C_{k} .
$$

Since the factor $\left(\frac{2^{n+1}\left(S+b_{k}\right)^{n}}{(2 S)^{n}}\right)^{\frac{1}{p}}$ is bounded for sufficiently large $S$, expression 15 does not exceed some constant and therefore, each expression

$$
\frac{1}{(2 S)^{n}} \int_{\Omega(-S I, 2 S I)}\left|f_{1}(x+t)-f_{1}\left(t+h_{k}\right)\right|^{p} d t
$$

is bounded for sufficiently large $S$. 
Passing to the limit, we arrive at the following inequality

$$
\begin{gathered}
\varlimsup_{S \rightarrow \infty} \frac{1}{(2 T)^{n}} \sum_{k=1}^{N} \int_{B_{k}} \frac{1}{(2 S)^{n}} \int_{\Omega(-S I, 2 S I)}\left|f_{1}(t+x)-f_{1}\left(t+h_{k}\right)\right|^{p} d t d x \\
\leqslant \frac{1}{(2 T)^{n}} \sum_{k=1}^{N} \int_{B_{k}} D_{B^{p}}^{p}\left[f_{1}(t+x), f_{1}\left(t+h_{k}\right)\right] d x .
\end{gathered}
$$

We apply inequality $10 p$. Since $\mu\left(B_{k}\right)<\delta, k=\overline{1, N}$, then $\left|(x+t)-\left(t+h_{k}\right)\right|<\delta$ holds $\forall x \in B_{k}$. Hence, for each $k=\overline{1, N}$ and each $x \in B_{k}$ the inequlaity

$$
D_{B^{p}}^{p}\left[f_{1}(t+x), f_{1}\left(t+h_{k}\right)\right]<\varepsilon^{p}
$$

holds true. Therefore, expression (16) does not exceed

$$
\frac{1}{(2 T)^{n}} \sum_{k=1}^{N} \int_{B_{k}} \varepsilon^{p} d x=\varepsilon^{p}
$$

that yields (13).

Thus, we have

$$
D_{B^{p}}\left[\frac{1}{(2 T)^{n}} \int_{\Omega(-T I, 2 T I)} f_{1}(x+t) d x, \sigma\left(f_{1}\right)\right] \leqslant \varepsilon
$$

Then

$D_{B^{p}}\left[\sigma\left(f_{1}\right), 1\right] \leqslant D_{B^{p}}\left[\frac{1}{(2 T)^{n}} \int_{\Omega(-T I, 2 T I)} f_{1}(x+t) d x, \sigma\left(f_{1}\right)\right]+D_{B^{p}}\left[\frac{1}{(2 T)^{n}} \int_{\Omega(-T I, 2 T I)} f_{1}(x+t) d x, 1\right]$.

Let us check that

$$
D_{B^{p}}\left[\frac{1}{(2 T)^{n}} \int_{\Omega(-T I, 2 T I)} f_{1}(x+t) d x, 1\right]<\varepsilon .
$$

It follows from inequality (9) that

$$
\left|\frac{1}{(2 T)^{n}} \int_{\Omega(-T I, 2 T I)} f_{1}(x+t) d x-1\right|^{p} \leqslant \varepsilon^{p} .
$$

Employing (18) we write the obvious chain of inequalities

$$
\begin{aligned}
D_{B^{p}} & {\left[\frac{1}{(2 T)^{n}} \int_{\Omega(-T I, 2 T I)} f_{1}(x+t) d x, 1\right] } \\
& =\left(\varlimsup_{S \rightarrow \infty} \frac{1}{(2 S)^{n}} \int_{\Omega(-S I, 2 S I)}\left|\frac{1}{(2 T)^{n}} \int_{\Omega(-T I, 2 T I)} f_{1}(t+x) d x-1\right|^{p} d t\right)^{\frac{1}{p}} \\
& \leqslant\left(\varlimsup_{S \rightarrow \infty} \frac{1}{(2 S)^{n}}(2 S)^{n} \varepsilon^{p}\right)^{\frac{1}{p}}=\varepsilon .
\end{aligned}
$$

It implies $D_{B^{p}}\left[\sigma\left(f_{1}\right), 1\right]<2 \varepsilon$. 
We return back to the previous notation and let $A_{k}:=\frac{\alpha \mu\left(B_{k}\right) e^{-i\left\langle\lambda, h_{k}\right\rangle}}{(2 T)^{n}}$ to obtain

$$
D_{B^{p}}\left[\sum_{k=1}^{N} A_{k} f\left(t+h_{k}\right), e^{i\langle\lambda, t\rangle}\right]<2 \varepsilon .
$$

Since $\varepsilon$ is arbitrary, we obtain that $\lambda \in \operatorname{sp}_{B} f$.

4) We consider the case $D=D_{U}$.

Let $\lambda \in \operatorname{sp} f$ and $f_{1}(t)=\frac{1}{\alpha} f(t) e^{-i\langle\lambda, t\rangle}$, where $\alpha=M\left\{f(t) e^{-i\langle\lambda, t\rangle}\right\}, \alpha \in \mathbb{R} \backslash\{0\}$. Then there exists the limit $\lim _{t \rightarrow \infty} \frac{1}{T^{n}} \int_{\Omega(0, T I)} f_{1}(x+t) d x=1$ uniformly in parameter $t \in \mathbb{R}^{n}$. Thus, for each $\varepsilon>0$ there exists a number $T_{0}=T_{0}(\varepsilon)$ independent of $t$ such that for each $T>T_{0}$ the inequality

$$
\left|\frac{1}{T^{n}} \int_{\Omega(0, T I)} f_{1}(x+t) d x-1\right|<\varepsilon
$$

holds true. Let us show that for each $\varepsilon>0$ there exists an integral sum corresponding to the above integral, which approximates this integral uniformly in $t \in \mathbb{R}^{n}$. Since $f_{1}(t)$ is uniform a.p. function, it is uniformly continuous in $\mathbb{R}^{n}$, i.e., for each $\varepsilon>0$ there exists $\delta=\delta(\varepsilon)$ such that if $|h|<\delta$, then for each $t \in \mathbb{R}^{n}$

$$
\left|f_{1}(t+h)-f_{1}(t)\right|<\varepsilon .
$$

As above, let $\left\{B_{k}\right\}_{k=1}^{N}$ be an arbitrary partition of squared beam $\Omega(0, T I)$ such that $\operatorname{diam}\left(\mathrm{B}_{\mathrm{k}}\right)<\delta(k=\overline{1, N})$, where $\delta$ is taken from 20. . It is obvious that $\sum_{k=1}^{N} \mu\left(B_{k}\right)=T^{n}$, where $\mu$ is the Lebesgue measure in $\mathbb{R}^{n}$. Let $\left\{h_{k}\right\}_{k=1}^{N}$ be an arbitrary set of points in $\mathbb{R}^{n}$ such that $h_{k} \in B_{k}, k=\overline{1, N}$. Then the integral sum associated with this partition reads as

$$
\sigma\left(f_{1}\right)=\frac{1}{T^{n}} \sum_{k=1}^{N} f_{1}\left(t+h_{k}\right) \mu\left(B_{k}\right) .
$$

We estimate $\left|\frac{1}{T^{n}} \int_{\Omega(0, T I)} f_{1}(t+x) d x-\sigma\left(f_{1}\right)\right|$ and obtain

$$
\begin{aligned}
& \left|\frac{1}{T^{n}} \int_{\Omega(0, T I)} f_{1}(t+x) d x-\frac{1}{T^{n}} \sum_{k=1}^{N} f_{1}\left(t+h_{k}\right) \mu\left(B_{k}\right)\right| \\
& =\frac{1}{T^{n}}\left|\sum_{k=1}^{N} \int_{B_{k}}\left(f_{1}(t+x)-f_{1}\left(t+h_{k}\right)\right) d x\right| \\
& \leqslant \frac{1}{T^{n}} \sum_{k=1}^{N} \int_{B_{k}}\left|f_{1}(t+x)-f_{1}\left(t+h_{k}\right)\right| d x .
\end{aligned}
$$

We observe that if $\operatorname{diam}\left(\mathrm{B}_{\mathrm{k}}\right)<\delta(k=\overline{1, N})$, then for each $x \in B_{k}$ we have $\left|(x+t)-\left(t+h_{k}\right)\right|=$ $\left|x-h_{k}\right|<\delta$. By 20 it implies

$$
\left|f_{1}(x+t)-f_{1}\left(t+h_{k}\right)\right|<\varepsilon
$$

Employing 22 for 21 , we obtain that 21 does not exceed $\frac{1}{T^{n}} \varepsilon \sum_{k=1}^{N} \mu\left(B_{k}\right)=\frac{1}{T^{n}} \varepsilon T^{n}=\varepsilon$. 
Thus, for each $\varepsilon>0$ there exists integral sum $\sigma\left(f_{1}\right)$ associated with a partition independent of $t$ such that

$$
\left|\frac{1}{T^{n}} \int_{\Omega(0, T I)} f_{1}(t+x) d x-\sigma\left(f_{1}\right)\right|<\varepsilon\left(\forall t \in \mathbb{R}^{n}\right) .
$$

It follows from inequalities 19 and 23 that

$$
\left|\sigma\left(f_{1}\right)-1\right| \leqslant\left|\sigma\left(f_{1}\right)-\frac{1}{T^{n}} \int_{\Omega(0, T I)} f_{1}(t+x) d x\right|+\left|\frac{1}{T^{n}} \int_{\Omega(0, T I)} f_{1}(t+x) d x-1\right|<2 \varepsilon,
$$

or, returning back to the previous notations, we have

$$
\left|\frac{\alpha}{T^{n}} \sum_{k=1}^{N} f\left(t+h_{k}\right) e^{-i\langle\lambda, t\rangle} e^{-i\left\langle\lambda, h_{k}\right\rangle} \mu\left(B_{k}\right)-1\right|<2 \varepsilon .
$$

We denote $A_{k}:=\frac{\alpha \mu\left(B_{k}\right) e^{-i\left\langle\lambda, h_{k}\right\rangle}}{T^{n}}$ to obtain

$$
\left|\sum_{k=1}^{N} A_{k} f\left(t+h_{k}\right)-e^{i\langle\lambda, t\rangle}\right|<2 \varepsilon
$$

Hence, $\lambda \in \operatorname{sp}_{B} f$.

Thus, the inclusion $\operatorname{sp} f \subset \operatorname{sp}_{B} f$ is proved for the cases $D=D_{U}, D_{S_{l}^{p}}, D_{W^{p}}, D_{B^{p}}$.

Let us prove the inclusion $\operatorname{sp}_{B} f \subset \operatorname{sp} f$. We shall argue by contradiction.

1) We consider the case $D=D_{W^{p}}$.

Let $\lambda \in \operatorname{sp}_{B} f \backslash \operatorname{sp} f$. Employing the properties of the mean, we have the following chain of equivalent statements

$$
\begin{aligned}
\lambda \notin \operatorname{sp} f \Leftrightarrow M\left\{f(t) e^{-i\langle\lambda, t\rangle}\right\}=0 & \Leftrightarrow M\left\{f(t+x) e^{-i\langle\lambda, t+x\rangle}\right\}=0 \\
& \Leftrightarrow M\left\{f(t+x) e^{-i\langle\lambda, t\rangle}\right\}=0 .
\end{aligned}
$$

Since $\lambda \in \operatorname{sp}_{B} f$, then $\forall \varepsilon>0 \exists\left\{A_{k}\right\}_{k=1}^{m} \subset \mathbb{R} \exists\left\{h_{k}\right\}_{k=1}^{m} \subset \mathbb{R}^{n}$ :

$$
D_{W^{p}}\left[e^{i\langle\lambda, x\rangle}, \sum_{k=1}^{m} A_{k} f\left(x+h_{k}\right)\right]<\varepsilon .
$$

In other words, $\forall \varepsilon>0 \exists\left\{A_{k}\right\}_{k=1}^{m} \subset \mathbb{R} \exists\left\{h_{k}\right\}_{k=1}^{m} \subset \mathbb{R}^{n}$ :

$$
\lim _{l \rightarrow \infty} \sup _{t \in \mathbb{R}^{n}}\left(\frac{1}{l^{n}} \int_{\Omega(t, l I)}\left|\sum_{k=1}^{m} A_{k} f\left(h_{k}+x\right)-e^{i\langle\lambda, x\rangle}\right|^{p} d x\right)^{\frac{1}{p}}<\varepsilon .
$$

In what follows we fix $\varepsilon$ and we assume $\varepsilon<\frac{1}{2}$. It yields the existence of $l<\infty$ such that

$$
\sup _{t \in \mathbb{R}^{n}}\left(\frac{1}{l^{n}} \int_{\Omega(t, l I)}\left|\sum_{k=1}^{m} A_{k} f\left(h_{k}+x\right)-e^{i\langle\lambda, x\rangle}\right|^{p} d x\right)^{\frac{1}{p}}<\varepsilon .
$$

This is why for each $t \in \mathbb{R}^{n}$ the inequality

$$
\left(\frac{1}{l^{n}} \int_{\Omega(t, l I)}\left|\sum_{k=1}^{m} A_{k} f\left(h_{k}+x\right)-e^{i\langle\lambda, x\rangle}\right|^{p} d x\right)^{\frac{1}{p}}<\varepsilon
$$


holds true or

$$
\left(\frac{1}{l^{n}} \int_{\Omega(t, l I)}\left|\sum_{k=1}^{m} A_{k} f\left(h_{k}+x\right) e^{-i\langle\lambda, x\rangle}-1\right|^{p} d x\right)^{\frac{1}{p}}<\varepsilon .
$$

We make a change of variables and get

$$
\left(\frac{1}{l^{n}} \int_{\Omega(0, l I)}\left|\sum_{k=1}^{m} A_{k} f\left(h_{k}+x+t\right) e^{-i\langle\lambda, t+x\rangle}-1\right|^{p} d x\right)^{\frac{1}{p}}<\varepsilon
$$

Applying Hölder inequality to 25, we have

$$
\begin{aligned}
& \frac{1}{l^{n}} \int_{\Omega(0, l I)}\left|\sum_{k=1}^{m} A_{k} f\left(h_{k}+x+t\right) e^{-i\langle\lambda, t+x\rangle}-1\right| d x \\
& \leqslant\left(\frac{1}{l^{n}} \int_{\Omega(0, l I)}\left|\sum_{k=1}^{m} A_{k} f\left(h_{k}+x+t\right) e^{-i\langle\lambda, t+x\rangle}-1\right|^{p} d x\right)^{\frac{1}{p}}<\varepsilon .
\end{aligned}
$$

We integrate the left hand side of 26$)$ over $\Omega(0, T I)$, where $T>T_{0}$. Then we switch the integration order to obtain

$$
\frac{1}{l^{n}} \int_{\Omega(0, l I)} \frac{1}{T^{n}} \int_{\Omega(0, T I)}\left|\sum_{k=1}^{m} A_{k} f\left(h_{k}+x+t\right) e^{-i\langle\lambda, x+t\rangle}-1\right| d t d x<\varepsilon .
$$

Then

$$
\frac{1}{l^{n}} \int_{\Omega(0, l I)}\left|\frac{1}{T^{n}} \int_{\Omega(0, T I)}\left(\sum_{k=1}^{m} A_{k} f\left(h_{k}+x+t\right) e^{-i\langle\lambda, x+t\rangle}-1\right) d t\right| d x<\varepsilon .
$$

or

$$
\frac{1}{l^{n}} \int_{\Omega(0, l I)}\left|\sum_{k=1}^{m} A_{k} e^{-i\langle\lambda, x\rangle} \frac{1}{T^{n}} \int_{\Omega(0, T I)} f\left(h_{k}+x+t\right) e^{-i\langle\lambda, t\rangle} d t-1\right| d x<\varepsilon .
$$

Employing the definition of the mean, by (24) we obtain

$$
\forall \varepsilon_{1}>0 \exists T_{0}>0 \forall T>T_{0}:\left|\frac{1}{T^{n}} \int_{\Omega(0, T I)} f\left(h_{k}+x+t\right) e^{-i\langle\lambda, t\rangle} d t\right|<\varepsilon_{1},
$$

where we choose $\varepsilon_{1}=\frac{\varepsilon}{\sum_{k=1}^{m}\left|A_{k}\right|}$.

By (28) we have

$$
\begin{aligned}
& \left|\sum_{k=1}^{m} A_{k} e^{-i\langle\lambda, x\rangle} \frac{1}{T^{n}} \int_{\Omega(0, T I)} f\left(h_{k}+x+t\right) e^{-i\langle\lambda, t\rangle} d t\right| \\
& \leqslant \sum_{k=1}^{m}\left|A_{k}\right| \cdot\left|\frac{1}{T^{n}} \int_{\Omega(0, T I)} f\left(h_{k}+x+t\right) e^{-i\langle\lambda, t\rangle} d t\right|<\varepsilon_{1} \sum_{k=1}^{m}\left|A_{k}\right|=\varepsilon .
\end{aligned}
$$


This is why

$$
\frac{1}{l^{n}} \int_{\Omega(0, l I)}\left|\sum_{k=1}^{m} A_{k} e^{-i\langle\lambda, x\rangle} \frac{1}{T^{n}} \int_{\Omega(0, T I)} f\left(h_{k}+x+t\right) e^{-i\langle\lambda, t\rangle} d t-1\right| d x>1-\varepsilon .
$$

The obtained inequality contradicts $(27)$ and the choice of $\varepsilon$. It proves the inclusion $\operatorname{sp}_{B} f \subset \operatorname{sp} f$ in the considered case.

2) It is easy to see that in the case $D=D_{S^{p}}$ the proof becomes simpler. It is sufficient to choose $l=1$ and to reproduce literally the main part of the proof.

3) We consider the case $D=D_{B^{p}}$. We note that (24) still holds.

Let $\lambda \in \operatorname{sp}_{B} f \backslash \operatorname{sp} f$, then $\forall \varepsilon>0 \exists\left\{A_{k}\right\}_{k=1}^{m} \subset \mathbb{R} \exists\left\{h_{k}\right\}_{k=1}^{m} \subset \mathbb{R}^{n}$ :

$$
D_{B^{p}}\left[e^{i\langle\lambda, x\rangle}, \sum_{k=1}^{m} A_{k} f\left(x+h_{k}\right)\right]<\varepsilon
$$

In other words, $\forall \varepsilon>0 \exists\left\{A_{k}\right\}_{k=1}^{m} \subset \mathbb{R} \exists\left\{h_{k}\right\}_{k=1}^{m} \subset \mathbb{R}^{n}$ :

$$
\left(\lim _{T \rightarrow \infty} \frac{1}{(2 T)^{n}} \int_{\Omega(-T I, 2 T I)}\left|\sum_{k=1}^{m} A_{k} f\left(h_{k}+x\right)-e^{i\langle\lambda, x\rangle}\right|^{p} d x\right)^{\frac{1}{p}}<\varepsilon,
$$

and we still assume that $\varepsilon<\frac{1}{2}$.

Applying Hölder inequality, we have

$$
\varlimsup_{T \rightarrow \infty} \frac{1}{(2 T)^{n}}\left|\int_{2(-T I, 2 T I)}\left(\sum_{k=1}^{m} A_{k} f\left(h_{k}+x\right)-e^{i\langle\lambda, x\rangle}\right)\right| d x<\varepsilon .
$$

We then obtain

$$
\varlimsup_{T \rightarrow \infty}\left|\sum_{k=1}^{m} A_{k} \frac{1}{(2 T)^{n}} \int_{\Omega(-T I, 2 T I)} f\left(h_{k}+x\right) e^{-i\langle\lambda, x\rangle} d x-1\right|<\varepsilon .
$$

On the other hand, employing the definition of the mean, by 24 we obtain

$$
\forall h_{k}>0 \exists T_{k}>0 \forall T>T_{k}:\left|\frac{1}{(2 T)^{n}} \int_{\Omega(-T I, 2 T I)} f\left(h_{k}+t\right) e^{-i\langle\lambda, t\rangle} d t\right|<\varepsilon_{1},
$$

where we choose $\varepsilon_{1}=\frac{\varepsilon}{\sum_{k=1}^{m}\left|A_{k}\right|}$.

By 31 for all $T>\max _{k} T_{k}$ we have

$$
\begin{aligned}
& \left|\sum_{k=1}^{m} A_{k} \frac{1}{(2 T)^{n}} \int_{\Omega(-T I, 2 T I)} f\left(h_{k}+x\right) e^{-i\langle\lambda, t\rangle} d x\right| \\
& \leqslant \sum_{k=1}^{m}\left|A_{k}\right| \cdot\left|\frac{1}{T^{n}} \int_{\Omega(-T I, 2 T I)} f\left(h_{k}+x\right) e^{-i\langle\lambda, x\rangle} d x\right|<\varepsilon_{1} \sum_{k=1}^{m}\left|A_{k}\right|=\varepsilon .
\end{aligned}
$$

This is why

$$
\left|\sum_{k=1}^{m} A_{k} \frac{1}{(2 T)^{n}} \int_{\Omega(-T I, 2 T I)} f\left(h_{k}+x\right) e^{-i\langle\lambda, x\rangle} d x-1\right|>1-\varepsilon .
$$


The obtained inequality contradicts (30) and the choice of $\varepsilon$ that proves the inclusion $\operatorname{sp}_{B} f \subset$ sp $f$ in the considered case.

4) In the case of uniform metric, by $(24)$ and inequality $|M\{f(t)\}| \leqslant \sup _{t \in \mathbb{R}^{n}}|f(t)|$, instead of (25)-(27) we obtain: $\forall \varepsilon>0 \quad \exists\left\{A_{k}\right\}_{k=1}^{m} \subset \mathbb{R} \quad \exists\left\{h_{k}\right\}_{k=1}^{m} \subset \mathbb{R}^{n}$ :

$$
\left|M\left\{\sum_{k=1}^{m} A_{k} f\left(t+h_{k}\right) e^{-i\langle\lambda, t\rangle}-1\right\}\right|<\varepsilon .
$$

Then

$$
\left|\sum_{k=1}^{m} A_{k} M\left\{f\left(t+h_{k}\right) e^{-i\langle\lambda, t\rangle}\right\}-1\right|<\varepsilon
$$

that contradicts to the choice of $\varepsilon$. The proof is complete.

\section{BIBLIOGRAPHY}

1. A.S. Besicovitch. Almost periodic function. Cambridge Univ. Press, Cambridge (1932).

2. A. Beurling. On the spectral synthesis of bounded functions // Acta Math. 81:1, 225-238 (1948).

3. S.Yu. Favorov, N. Girya. A multidimensional version of Levin's secular constant Theorem and its applications // Zhurn. Matem. Fiz. Anal. Geom. 3:3, 365-377 (2007). (in Russian).

4. L.H. Loomis. The spectral Characterization of a class of almost periodic functions // Ann. Math. Second Ser. 72:2, 362-368 (1960).

5. L.I. Ronkin. Almost periodic distributions in tube domains // Zap. Nauchn. Sem. POMI. 247. 210-236 (1997). [J. Math. Sci. 101:3, 3172-3189 (2000).]

6. H. Bohr. Fastperiodische Funktionen. Ergebnisse der Mathematik und ihrer Grenzgebiete. 1:5. Julius Springer, Berlin (1932).

7. S.Yu. Favorov, N.P. Girya. Asymptotic properties of holomorphic almost periodic functions // Matematychni Studii. 25:2, 191-201 (2006). (in Russian).

8. N.P. Girya. Almost periodic in the Besicovitch sense function with spectrum in a cone // Matematychni Studii. 27:2, 163-173 (2007). (in Russian).

9. B.M. Levitan. Almost periodic functions. Gostekhizdat, Moscow (1953). (in Russian).

10. V.V. Stepanov. On a class of almost periodic functions // Dokl. AN SSSR. 64:3, 297-300 (1949). (in Russian).

Natalia Petrovna Girya,

V. N. Karazin Kharkiv National University

Svobody av. 4,

61022, Kharkiv, Ukraine

E-mail: n_girya@mail.ru

Sergei Yurievich Favorov,

V. N. Karazin Kharkiv National University

Svobody av. 4,

61022, Kharkiv, Ukraine

E-mail: sfavorov@gmail.com 